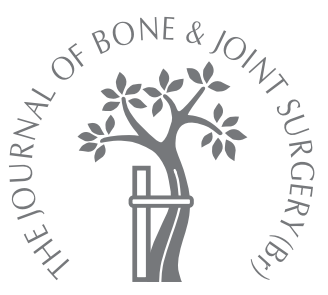

W.-C. Lee, J. H. Yoo, J.-S. Moon

From Seoul Paik Hospital, Seoul, Korea

W.-C. Lee, Md, PhD,

Professor

In.-S. Moon, MD, Orthopaedic

Surgeon

Department of Orthopaedic

Surgery

Seoul Paik Hospital, College of Medicine, Inje University, 85

2-ga, Jeo-dong, Jung-Gu,

Seoul 100-032, Korea.

들. J. Yoo, MD, Orthopaedic Surgeon

Department of Orthopaedic Surgery

Soonchunhyang Hospital, College of Medicine, Soonchunhyang University, 1174 Jung-Dong, Wonmi-Gu, Bucheon-Si, Gyeonggi-Do 420767, Korea.

Correspondence should be sent to Professor W.-C. Lee; e-mail: 1wsk980@unitel.co.kr

(C)2009 British Editorial Society of Bone and Joint Surgery doi:10.1302/0301-620X.91B11. $22169 \$ 2.00$

$J$ Bone Joint Surg [Br] 2009;91-B:1472-7. Received 8 December 2008; Accepted after revision 1 July 2009

\title{
Lengthening of fourth brachymetatarsia by three different surgical techniques
}

We carried out a retrospective study to assess the clinical results of lengthening the fourth metatarsal in brachymetatarsia in 153 feet of 106 patients (100 female, six males) using three different surgical techniques. In one group lengthening was performed by one-stage intercalary bone grafting secured by an intramedullary Kirschner-wire (45 feet, 35 patients). In the second group lengthening was obtained gradually using a mini-external fixator after performing an osteotomy with a saw (59 feet, 39 patients) and in the third group lengthening was achieved in a gradual manner using a mini-external fixator after undertaking an osteotomy using osteotome through pre-drilled holes (49 feet, 32 patients). The mean age of the patients was 26.3 years (13 to 48 ). Pre-operatively, the fourth ray of the bone-graft group was longer than that of other two groups $(p<0.000)$. The clinical outcome was compared in the three groups. The mean follow-up was 22 months (7 to 55).

At final follow-up, the mean lengthening in the bone-graft group was $13.9 \mathrm{~mm}$ (3.5 to 23.0, 27.1\%) which was less than that obtained in the saw group with a mean of $17.8 \mathrm{~mm}$ $(7.0$ to $33.0,29.9 \%)$ and in the pre-drilled osteotome group with a mean of $16.8 \mathrm{~mm}$ (6.5 to 28.0, 29.4\%, $p=0.001$ ). However, the mean time required for retention of the fixation in the bone-graft group was the shortest of the three groups. Patients were dissatisfied with the result for five feet $(11.1 \%)$ in the bone-graft group, eight $(13.6 \%)$ in the saw group and none in the pre-drilled osteotomy group $(p<0.000)$. The saw group included eight feet with failure of bone formation after surgery. Additional operations were performed in $\mathbf{2 0}$ feet because of stiffness ( $n=7$, all groups), failure of bone formation ( $n=4$, saw group), skin maceration ( $n=4$, bone-graft group), malunion ( $n=4$, bone-graft and saw groups) and breakage of the external fixator ( $n=1$, saw group).

We conclude that the gradual lengthening by distraction osteogenesis after osteotomy using an osteotome produces the most reliable results for the treatment of fourth brachymetatarsia.

Brachymetatarsia is a rare congenital deformity which occurs most commonly in the fourth metatarsal. ${ }^{1-3}$ It can also occur iatrogenically ${ }^{4,5}$ or traumatically. ${ }^{2,6,7}$ Patients with brachymetatarsia often exclude themselves from social activities because of the appearance of their foot. The distress felt by affected patients tends to be greater in Asian countries where the feet are generally exposed indoors.

Both one-stage intercalary bone grafting and gradual lengthening by distraction osteogenesis have been reported to achieve a satisfactory outcome. ${ }^{6,8-10}$ However, in the former, an additional incision is required at the iliac crest with possible accompanying complications and the extent of lengthening is limited by soft-tissue tension and ischaemic changes. The principal disadvantages of gradual lengthening are a longer duration until bone consolidation and the risk of insufficient formation of new bone. Only one study has compared the results of one-stage intercalary bone grafting with gradual lengthening in a small number of patients. ${ }^{9}$ For this reason it is not clear whether one method of lengthening is better than the other.

We have treated fourth-ray brachymetatarsia using three different techniques and have compared the results.

\section{Patients and Methods}

Our study included 153 feet in 106 patients (100 female, six male) who had been treated between 2001 and 2007. In 47 patients the deformity was bilateral. The mean age of the patients was 26.3 years (13 to 48 ) with a mean follow-up of 22 months (7 to 55). The study was confined to patients with congenitally 
short fourth metatarsals, and those with multiple brachymetatarsia and brachymetatarsia as a result of trauma, were excluded. All the procedures were performed by one surgeon (WCL).

The study comprised three groups of patients one of which received one-stage lengthening by intercalary bone grafting and the other two by gradual lengthening. The last two differed according to whether the osteotomy was fashioned with a saw or through a predrilled site. The advantages and disadvantages of the different procedures were discussed with the patients pre-operatively so that they could decide for themselves which method was to be used. However, the decision to saw or to divide a predrilled osteotomy was made following a change in the technique of osteotomy. Initially, the osteotomy for gradual lengthening was performed using a microsaw, but after eight feet (six patients) failed to achieve osteogenesis, it was then performed with a small osteotome after multiple drillholes had been made at the cortex. Therefore earlier patients lengthened by gradual lengthening were categorised into a saw group and later patients into a predrilled osteotomy group.

A total of 45 feet in 35 patients had one-stage lengthening by intercalary bone grafting and 108 feet in 71 patients had gradual lengthening with a mono-plane minifixator (Seoul Meditech, Seoul, Korea). Of the latter group, 59 feet in 39 patients were in the saw group and 49 feet in 32 patients in the predrilled osteotomy group. There was a normal control group consisting of 37 contralateral feet of patients with unilateral brachymetatarsia.

Pre-operatively, age, gender, and bilateralism were compared in the three groups and the control group. The length of the proximal phalanx of the fourth toe was compared with that of the third toe and the relative length of the fourth metatarsal with that of the second metatarsal expressed as a percentage.

The lengthening of the fourth metatarsal was calculated from the difference between the pre- and post-operative lengths. The length was defined as the distance between the points at which the midaxial line crossed the proximal and distal articular surfaces on a weight-bearing dorsoplantar radiograph of the foot. The percentage increase was calculated as the lengthening obtained divided by the preoperative length. A healing index was determined by dividing the duration until removal of Kirschner (K-)wires or the external fixator in weeks by the lengthened distance in centimetres. The American Orthopaedic Foot and Ankle Society (AOFAS) lesser toe metatarsophalangeal-interphalangeal score, ${ }^{11}$ satisfaction of patient and willingness to undergo the same surgery were obtained at the last follow-up. Complications and re-operations were reviewed.

\section{Operative technique}

Intercalary bone grafting. A dorsal longitudinal skin incision was made over the fourth metatarsal. After subperiosteal dissection, a transverse osteotomy was made with a microsaw at the level of the mid-shaft. A $1.6 \mathrm{~mm} \mathrm{~K}$ wire was then passed distally along the intramedullary

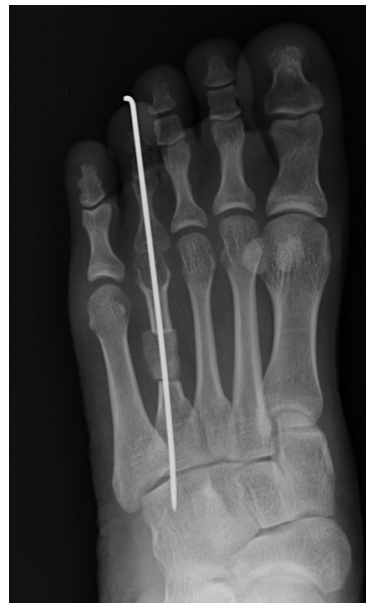

Fig. 1a

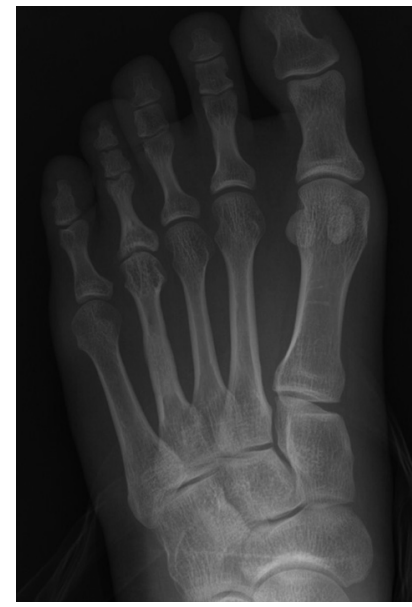

Fig. 1b
Dorsoplantar radiographs of a foot showing a) the lengthened fourth metatarsal using intercalary iliac-bone grafting longitudinally fixed by a Kirschner-wire and b) four years after surgery.

canal from the osteotomy and then continued distally through the phalanges to the tip of the phalanx. The wire was retrieved distally and drawn further distally until its proximal end was located just distal to the site of the osteotomy. A lamina spreader was inserted in the site and distraction performed to stretch the soft tissues for 20 to 30 minutes as suggested in previous reports. ${ }^{8,9}$ At this stage a tricortical iliac-bone graft was obtained and inserted tightly between the surfaces. The K-wire already in the distal fragment was then drilled through the graft and advanced proximally into the cuboid (Fig. 1). A short-leg splint (below-knee slab) was applied post-operatively and weight-bearing on the heel was allowed. In order to prevent maceration of soft tissue at the flexion crease of the base of the toe, dry gauze was placed deeply in the flexion crease.

Application of an external fixator and osteotomy of the metatarsal shaft. A dorsal longitudinal incision of $1.5 \mathrm{~cm}$ was made over the distal half of the metatarsal. The periosteum was reflected at the mid-shaft and the first minihalf-pin was inserted at the head-neck junction after predrilling with a $1.4 \mathrm{~mm} \mathrm{~K}$-wire. The second distal mini-half pin was placed in a similar manner under direct vision at the neck of the metatarsal using an appropriate placement guide (Fig. 2). The length of the external fixator was adjusted to about $3.0 \mathrm{~cm}$ to $3.5 \mathrm{~cm}$ from the most distal mini-half-pin to the most proximal mini-half-pin before placing the proximal mini-half-pins. Initially, $\mathrm{K}$-wires were used for the proximal pin sites. Fluoroscopy was used to confirm the position of the distal mini-half-pins and proximal K-wires. If the latter were correctly positioned in the proximal fragment they were exchanged for mini-half-pins. The external fixator was applied and the osteotomy made half way between the second and third mini-half-pins (Fig. 3 ). In the saw group, the osteotomy was made using a microsaw with cold-water irrigation to minimise thermal 


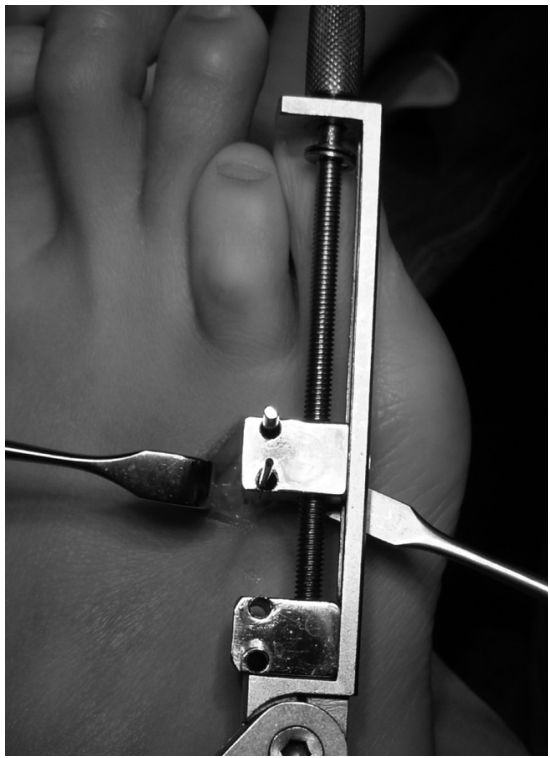

Fig. 2

Photograph showing that after placement of a guide with the first pin in the most distal hole, the second pin site is drilled using a $0.057 \mathrm{~mm}$ Kirschner-wire under direct vision in the neck of the metatarsal.

necrosis. In the predrilled osteotomy group, multiple drillholes were made at the site of the osteotomy which was completed using an osteotome. Subcutaneous tissue and skin were sutured.

All the patients were allowed to walk weight-bearing as tolerated from the first post-operative day. The patients returned at two weeks post-operatively for examination of the wound and training in the distraction technique. This was begun at a rate of $0.7 \mathrm{~mm} /$ day which represented one turn in the external fixator adjustor and divided into four quarter turns every six hours. They returned ten to 14 days later for confirmation of adequate distraction, and at an interval of three to four weeks later for radiological examination. Pin-care with antiseptics was recommended only after showering and no other regular pin-care was suggested. Gauze was tightly wrapped around the mini-halfpin to prevent movement between the skin and the pin. Pain from stretching of the soft tissues was experienced in almost all the patients during lengthening, and the distraction rate was lowered or discontinued for several days when severe pain occurred.

The fixator was removed in the outpatient clinic after maturation of callus after which full weight-bearing was allowed, but with no running for four weeks.

Statistical analysis. We examined clinical variables, the functional status and the outcome variable using descriptive and univariate statistics. For continuous variables, the mean, the SD and the $95 \%$ confidence interval (CI) were reported. For categorical variables, frequencies and percentages were reported. A chi-squared test was used to exam-

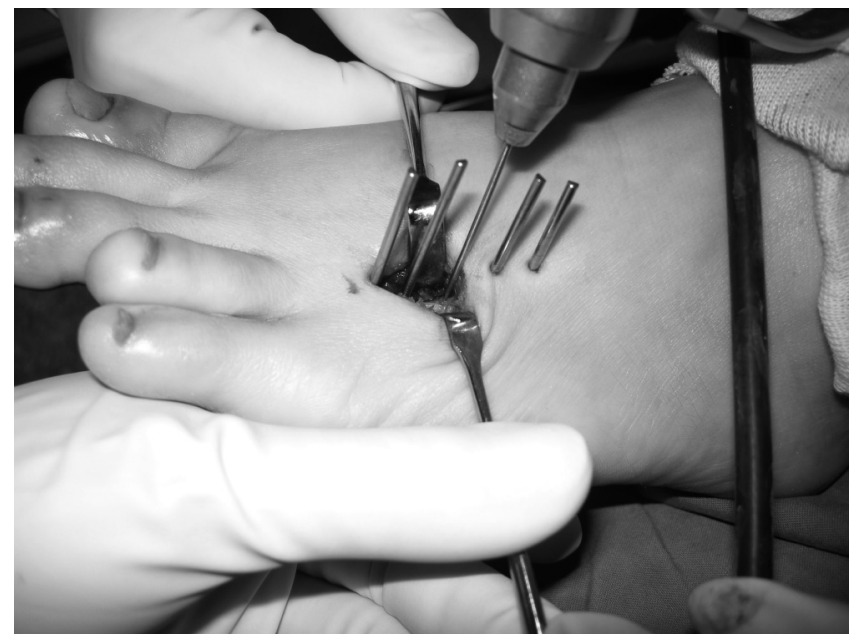

Fig. 3a

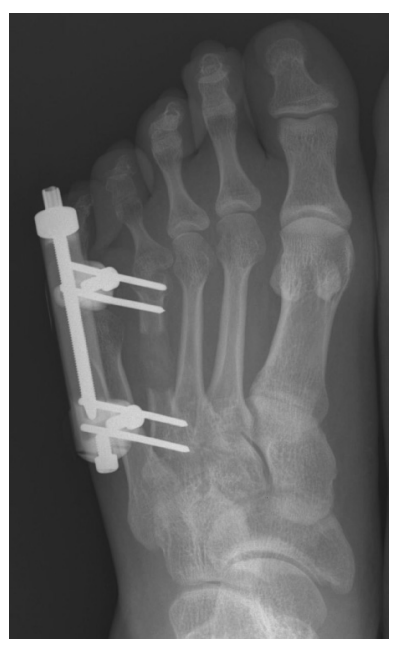

Fig. 3b

a) Photograph showing multiple holes made by a Kirschner-wire in the middle of the proximal and distal mini-half-pins and b) a dorsoplantar radiograph of the foot showing the lengthened fourth metatarsal with the external fixator.

ine the differences in the three groups in regard to gender, bilateralism, subjective satisfaction and willingness to undergo the same surgery.

One-way analysis of variance (ANOVA) was used to compare differences in age, shortness of the proximal phalanx of the third toe to the fourth toe, the percentage of the pre-operative length of the fourth metatarsal to the second metatarsal, the AOFAS lesser toe metatarsophalangealinterphalangeal score, the gain in length, the percentage of gain in length, the healing index and the duration of fixation in the three groups. The Tukey post hoc test was used for the determination of differences in the three groups. A two-tailed p-value $\leq 0.05$ was considered to be statistically significant. All statistical analyses were performed using SPSS version 10.0.7 software (SPSS Inc., Chicago,Illinois). 
Table I. Details of the patients in each group

\begin{tabular}{|c|c|c|c|c|}
\hline & $\begin{array}{l}\text { One-stage lengthening } \\
(n=35)\end{array}$ & $\begin{array}{l}\text { Distraction osteogenesis using saw } \\
(\mathrm{n}=39)\end{array}$ & $\begin{array}{l}\text { Distraction osteogenesis using osteotomy } \\
(\mathrm{n}=32)\end{array}$ & p-value \\
\hline Mean (SD) age in years & $28.4(6.1)$ & $25.5(5.8)$ & $25.1(5.4)$ & 0.041 \\
\hline Number of females (\%) & 32 (91.4) & $37 \quad(94.9)$ & $31 \quad(96.9)$ & 0.580 \\
\hline Number with bilaterality (\%) & $10(28.6)$ & $20 \quad(51.3)$ & $17 \quad(53.1)$ & 0.071 \\
\hline
\end{tabular}

Table II. Pre-operative radiological variables with mean values and 95\% confidence intervals in parentheses, compared with the control group

\begin{tabular}{|c|c|c|c|c|c|}
\hline & $\begin{array}{l}\text { One-stage lengthening } \\
(n=45)\end{array}$ & $\begin{array}{l}\text { Distraction osteogenesis } \\
\text { using saw }(\mathrm{n}=59)\end{array}$ & $\begin{array}{l}\text { Distraction osteogenesis } \\
\text { using osteotomy }(n=49)\end{array}$ & Control $(n=37)$ & p-value \\
\hline 4th $\mathrm{MT}^{*} / 2 \mathrm{nd} \mathrm{MT}(\%)$ & 72.7 (71.5 to 74.0$)$ & 70.2 (69.0 to 70.9$)$ & $70.1(69.0$ to 71.2$)$ & 91.0 (89.9 to 92.2$)$ & $<0.001^{\dagger}$ \\
\hline Shortness of 4 th toe $(\mathrm{mm})$ & $5.2(4.2$ to 6.2$)$ & $6.9(6.1$ to 7.7$)$ & $6.6(5.9$ to 7.3$)$ & $2.1(1.7$ to 2.6$)$ & $<0.001^{\ddagger}$ \\
\hline
\end{tabular}

* MT, metatarsal

$\dagger$ control vs brachymetatarsia groups

‡ bone-graft group vs distraction groups

Table III. Post-operative radiological variables with mean values and 95\% confidence intervals

\begin{tabular}{|c|c|c|c|c|}
\hline & $\begin{array}{l}\text { One-stage lengthening } \\
(n=45)\end{array}$ & $\begin{array}{l}\text { Distraction osteogenesis using saw } \\
(\mathrm{n}=51)^{*}\end{array}$ & $\begin{array}{l}\text { Distraction osteogenesis using osteotomy } \\
(\mathrm{n}=49)\end{array}$ & p-value \\
\hline Length gain (mm) & $13.9(12.6$ to 15.2$)$ & 17.8 (16.2 to 19.4$)$ & $16.8(15.4$ to 18.2$)$ & 0.001 \\
\hline Length gain (\%) & 27.1 (24.4 to 29.8 ) & 29.9 (27.3 to 32.5$)$ & 29.4 (27.4 to 31.4$)$ & 0.231 \\
\hline Healing index (wks/cm) & $8.2(7.0$ to 9.4$)$ & 9.0 (7.9 to 10.0$)$ & 8.4 (7.3 to 9.6$)$ & 0.617 \\
\hline Fixation time (wks) & $10.0(9.5$ to 10.6$)$ & 14.5 ( 13.5 to 15.5$)$ & $12.6(11.9$ to 13.2$)$ & $<0.001$ \\
\hline
\end{tabular}

* eight feet were excluded from the analysis of length gain and healing index because of failure of bone formation

\section{Results}

The baseline clinical details were similar in the three groups except for the mean age of the patients (Table I). For the percentage of the length of the fourth metatarsal to the second metatarsal and the shortness of the proximal phalanx of the third toe to the fourth toe, the control group differed from the brachymetatarsia groups and the bone-graft group from the distraction two groups (Table II).

The mean gain in length in the bone-graft group was $13.9 \mathrm{~mm}(3.5$ to $23.0,27.1 \%)$ which was less than that achieved in the saw group with a mean of $17.8 \mathrm{~mm}$ (7.0 to $33.0,29.9 \%$ ) and in the predrilled osteotomy group with a mean of $16.8 \mathrm{~mm}$ (6.5 to 28.0, 29.4\%; ANOVA, $\mathrm{p}<0.001$ ). More rapid bone healing in the bone-graft group was significant in terms of the mean fixation time (ANOVA, $\mathrm{p}=0.000$ ), but not when expressed as the healing index (ANOVA, $\mathrm{p}=0.617$; Table III).

The mean post-operative AOFAS score was similar in the groups (Table IV). In all, the patients were satisfied with the outcome for 102 feet $(67 \%)$, satisfied with reservation for 38 feet $(25 \%)$ and dissatisfied with their result for 13 feet $(8 \%)$. All the patients who were satisfied and satisfied with reservation said that they would undergo the same lengthening procedure again and all except one dissatisfied patient also would undergo the same procedure again. The proportion of dissatisfied patients was significantly fewer in the predrilled osteotomy group (chi-squared test, $\mathrm{p}<0.000$; Table IV). The dissatisfaction was due to an unsatisfactory gain in length in nine feet and painful stiffness of the metatarsophalangeal joint in four.

In one-stage lengthening, skin necrosis from maceration at the flexion skin fold of the metatarsophalangeal joint occurred in most cases. It was severe in four, which required removal of the longitudinal $\mathrm{K}$-wire and re-insertion of a transverse K-wire which enabled healing to continue without any further surgical procedures. Painful stiffness of the metatarsophalangeal joint was the cause of the patients' reservation and less than satisfactory results in 11 feet $(24.4 \%)$ in the bone-graft group, $12(20.3 \%)$ in the saw group and one $(2.0 \%)$ in the predrilled osteotomy group. Less lengthening than was cosmetically acceptable to the patient occurred in nine feet of the bone-graft group, 13 of the saw group which included eight feet with complete failure of bone formation and three in the predrilled osteotomy group. There was one case of an instrument-related complication in which the external fixator broke where the pin clamp connected to the rod.

Severe pin-track infection with intramedullary bone resorption occurred in one foot in the bone-graft group and three in the saw group. The infection resolved after removal of the K-wire or mini-half-pins and the administration of oral antibiotics.

Additional operations were performed in 20 feet including four which have already been mentioned above for skin 
Table IV. Post-operative clinical variables with mean values and $95 \%$ confidence intervals in parentheses

\begin{tabular}{|c|c|c|c|c|}
\hline & $\begin{array}{l}\text { One-stage lengthening } \\
(n=45)\end{array}$ & $\begin{array}{l}\text { Distraction osteogenesis } \\
\text { using saw }(n=59)\end{array}$ & $\begin{array}{l}\text { Distraction osteogenesis } \\
\text { using osteotomy }(n=49)\end{array}$ & p-value \\
\hline AOFAS $^{*}$ score & 86.0 (84.4 to 87.5$)$ & 85.5 (84.3 to 88.3 ) & 86.9 (85.6 to 88.3 ) & 0.508 \\
\hline \multicolumn{5}{|l|}{ Subjective satisfaction score } \\
\hline Satisfied & 25 & 32 & 45 & \\
\hline Satisfied with reservation & 15 & 19 & 4 & \\
\hline Dissatisfied & 5 & 8 & 0 & $<0.001$ \\
\hline \multicolumn{5}{|l|}{ Willingness } \\
\hline Yes & 41 & 51 & 49 & \\
\hline No & 4 & 8 & 0 & 0.032 \\
\hline
\end{tabular}

maceration and re-insertion of K-wires. Soft-tissue releases were performed in seven feet (three in the bone-graft group and four in in the saw group), osteotomy for angulation and stiffness in four (one in the bone-graft group and three in the saw group), bone grafting in four (saw group) and reapplication of external fixation with osteotomy at a different site than the original in one (saw group).

\section{Discussion}

Some reports ${ }^{3,8,12}$ have advocated one-stage lengthening in any patient with brachymetatarsia, but others have indicated distraction osteogenesis particularly in patients with short proximal phalanges or with greater shortening of the metatarsal. ${ }^{13,14}$

The main advantages of one-stage lengthening are the non-requirement for an external fixator, no possibility of insufficient bone formation and no necessity for manipulation of the external fixator after surgery. Disadvantages are the limited lengthening obtained, immobilisation of the metatarsophalangeal joint during consolidation of the graft and the necessity for harvesting a bone graft. Allograft instead of autogenous graft may be used without donor morbidity, but may lack the same capacity to heal. Any limitation of lengthening in this technique is mainly from softtissue tightness. The distance obtained in one-stage lengthening in our series was less than that in gradual lengthening, and we suspect that older age may be related to this observation. However, Kim et $\mathrm{al}^{6}$ reported that it is very difficult to lengthen more than $15 \mathrm{~mm}$ in one-stage lengthening even in younger patients. Ischaemia is another cause of limited gain in length by one-stage lengthening. When ischaemia persists after more than 15 minutes, removal of the longitudinal K-wire after transversely stabilising the divided metatarsal to adjacent metatarsals with $\mathrm{K}$-wires will help to restore blood flow. It may be reasonable to recommend one-stage lengthening in feet which require lengthening of less than $25 \%$ of the fourth metatarsal as we were able to lengthen that amount by one-stage lengthening.

The more common problem is the maceration of the plantar aspect of the metatarsophalangeal joint. Because of the plantar slope of the metatarsal, the fourth toe is immobilised in a plantar-flexed position, which promotes maceration between the skin folds.

The advantages of gradual lengthening are the ability to obtain greater length than that using one-stage intercalary bone grafting, immediate weight-bearing and the preservation of movement of the metatarsophalangeal joint. Distraction osteogenesis in small bones in the feet has been reported to be successful following the principles of Ilizarov, ${ }^{2,4,15}$ but with the disadvantage of regular adjustment of the external fixator, the possibility of insufficient bone formation and the risk of pin-track infection. In order to encourage adequate bone formation, careful osteotomy with the least soft-tissue and vascular damage and a secure external fixator are needed. Corticotomy in distraction osteogenesis should be performed using an osteotome ${ }^{16}$ to avoid heat damage and to protect the intramedullary vasculature. ${ }^{10,14}$ In our series, we thought that there would be minimal heat damage even though an osteotomy was performed with a microsaw without predrilling and that multiple predrilling would cause as much local heat damage. However, several failures in osteogenesis using this technique made us reconsider the technique of osteotomy, and the change to an osteotomy after predrilling multiple holes which markedly improved our results.

Our results of gradual distraction have provided lengthening comparable with that of previous reports. $1,2,9,10,13,14,17$ The healing index in the one-stage lengthening group and predrilled osteotome group was also comparable with that of previous reports. ${ }^{10,17,18}$

It has been stated that the main cause of the stiffness of the metatarsophalangeal joint is a percentage of lengthening greater than $40 \%$ of the pre-operative length. ${ }^{1,10}$ However, we think stiffness is a common feature even with lengthening of less than $40 \%$. In our series, the mean pre-operative length of the fourth metatarsal was about $70 \%$ of that of the second metatarsal which was about $90 \%$ in normal feet, and therefore lengthening of $30 \%$ would result in normal length. In addition, the proximal phalanx of the fourth toe was about $3 \mathrm{~mm}$ to $5 \mathrm{~mm}$ shorter than that of the normal foot. We 
counselled the patients to accept the short appearance as a result of shortness of the proximal phalanx to avoid stiffness and metatarsalgia. Therefore we did not aim to lengthen to $40 \%$ of the pre-operative length.

The pre-operative AOFAS score was 100 in all patients, and it was reduced after surgery. The main cause was pain at the plantar aspect of the proximal phalanx or at the metatarsophalangeal joint after prolonged standing or walking and decreased movement of this joint. However, most patients did not regard these symptoms as an important disability and therefore there is a discrepancy between the AOFAS score and measures of subjective satisfaction and willingness to undergo the operation again.

There were limitations to our study. First, it was not prospectively designed and therefore selection bias for the method of lengthening may have affected the result. The preoperative length of the fourth metatarsal and the proximal phalanx of the fourth toe in the bone-graft group were less short than those of the patients in other groups. Although the decision to choose the method of lengthening was made by the patients, it cannot be discounted that there may have been some influence by the surgeon towards one-stage lengthening for patients with less shortening. The length gain in one-stage lengthening was a mean of $13.9 \mathrm{~mm}$ which was less than that obtained in the gradual lengthening group. Secondly, the technique of osteotomy in gradual lengthening was changed after accumulating experience in the saw group. The result might have been better if all the osteotomies had been fashioned through pre-drilled holes.

In conclusion, gradual lengthening by distraction osteogenesis after osteotomy using an osteotome produces the most reliable results with longer lengthening within a predictable period.
No benefits in any form have been received or will be received from a commercial party related directly or indirectly to the subject of this article.

\section{References}

1. Masada K, Fujita S, Fuji T, Ohno H. Complications following metatarsal lengthening by callus distraction for brachymetatarsia. J Pediatr Orthop 1999;19:394-7.

2. Shim JS, Park SJ. Treatment of brachymetatarsia by distraction osteogenesis. J Pediatr Orthop 2006;26:250-4.

3. Urano Y, Kobayashi A. Bone-lengthening for shortness of the fourth toe. J Bone Joint Surg [Am] 1978;60-A:91-3.

4. Hurst JM, Nunley JA 2nd. Distraction osteogenesis for the shortened metatarsal after hallux valgus surgery. Foot Ankle Int 2007;28:194-8.

5. Sinclair GG, Shoemaker SK, Seibert SR. latrogenic brachymetatarsia. J Foot Surg 1991;30:580-4.

6. Kim HT, Lee SH, Yoo Cl, Kang JH, Suh JT. The management of brachymetatarsia. J Bone Joint Surg [Br] 2003;85-B:683-90.

7. Saxby T, Nunley A. Metatarsal lengthening by distraction osteogenesis: a report of two cases. Foot Ankle 1992;13:536-9.

8. Baek GH, Chung MS. The treatment of congenital brachymetatarsia by one-stage lengthening. J Bone Joint Surg [Br] 1998;80-B:1040-4.

9. Choi IH, Chung MS, Baek GH, Cho TJ, Chung CY. Metatarsal lengthening in congenital brachymetatarsia: one-stage lengthening versus lengthening by callotasis. $J$ Pediatr Orthop 1999;19:660-4

10. Song HR, Oh CW, Kyung HS, et al. Fourth brachymetatarsia treated with distraction osteogenesis. Foot Ankle Int 2003;24:706-11.

11. Kitaoka HB, Alexander IJ, Adelaar RS, et al. Clinical rating systems for the anklehindfoot, midfoot, hallux, and lesser toes. Foot Ankle Int 1994;15:349-53.

12. Pasternack WA. Brachymetatarsia: a unique surgical approach. J Am Podiatr Med Assoc 1988;78:415-18.

13. Schimizzi A, Brage M. Brachymetatarsia. Foot Ankle Clin 2004;9:555-70.

14. Levine SE, Davidson RS, Dormans JP, Drummond DS. Distraction osteogenesis for congenitally short lesser metatarsals. Foot Ankle Int 1995;16:196-200.

15. Wada A, Bensahel H, Takamura K, et al. Metatarsal lengthening by callus distraction for brachymetatarsia. J Pediatr Orthop B 2004;13:206-10.

16. Ilizarov G. The tension effect on the genesis and growth of tissues. Part I: the influence of stability of fixation and soft tissue preservation. Clin Orthop 1989;238:249-81.

17. Kawashima T, Yamada A, Ueda K, Harii K. Treatment of brachymetatarsia by callus distraction (callotasis). Ann Plast Surg 1994;32:191-9.

18. Ferrandez L, Yubero J, Usabiaga J, Ramos L. Congenital brachymetatarsia: three cases. Foot Ankle 1993;14:529-33. 